\title{
Requisites for Ultimate Energy Resolution EELS and Band Gap Measurements
}

\author{
E. Essers*, R. Höschen**, M. Matijevic*, G. Benner*, C. Koch** \\ * Carl Zeiss NTS GmbH, Carl-Zeiss-Straße 56, 73447 Oberkochen, Germany \\ ** Max Planck Institute for Metals Research, Heisenbergstraße 3, 70569 Stuttgart, Germany
}

The instrumental stability of the SESAMe microscope and resulting improvements in energy resolution and shape of the zero-loss peak (ZLP) define a new state of the art in electron energy-loss spectroscopy (EELS) using medium voltage $(200 \mathrm{kV})$ Transmission Electron Microscopes (TEMs). The excellent reproducibility of the width and shape of the ZLP is essential for reliable data acquisition and allows small spectral features close to (und usually hidden by) the ZLP to be revealed by deconvolution methods as well as a strongly improved energy resolution.

The development of monochromators in the last few years has strongly improved the energy resolution achievable in TEMs. Moreover the monochromators also reduce the tail of the ZLP, thereby strongly improving the possibilities for the measurement of small band gaps, defect states within them, and other low energy-loss spectral features. However, the impressing theoretically available energy resolution defined by slit width and aberrations of the monochromators requires a matching stability of other components of the microscope. Especially the stability requirements to the high voltage and filter current are demanding.

Stability improvements of these components at first lead to new energy resolution records at short acquisition times of one second or less, which are only of theoretical interest. Whereas practical applications with ultimate energy resolution usually need longer acquisition times, because of the weak signal intensity.

Subsequent further stability optimisation also strongly improves the energy resolution at longer acquisition times. If the shape of the ZLP thereby becomes not only narrow, but also reproducible and stable over long exposure times, then energy resolutions far below the width of the ZLP are made accessible by the use of deconvolution techniques (e.g.[1]). In combination with a monochromator this allows very high resolution EELS data to be acquired. Moreover also small spectral details, which are still hidden by the (already strongly reduced) tail of the ZLP, become visible.

We report on the Zeiss SESAMe microscope [2] which includes the CEOS electrostatic Omegatype monochromator $[3,4]$, Koehler illumination, a highly stable $200 \mathrm{kV}$ high voltage supply and the newly developed in-column MANDOLINE filter [5,6].

Figure 1 shows the EELS spectrum of the ZLP taken with $42 \mathrm{meV}$ monochromator energy-selection slit width and $10 \mathrm{~s}$ exposure time. The energy dispersion was $3.5 \mathrm{meV} / \mathrm{ch}$, and a $24 \mu \mathrm{m}$ square pixel CCD with a YAG single-crystal scintillator was utilized. The full width at half maximum (FWHM) is $87 \mathrm{meV}$. Three spectra where taken under these conditions during three minutes. All three of these spectra are shown in figure 2 in a logarithmic scale and coincide very well with one another. (The deviations of the three spectra in the range below $10^{-3.5}$ are due to shot noise.) Figure 2 also shows the steep drop of the ZLP to be $10^{-1}$ after $0.1 \mathrm{eV}, 10^{-2}$ after $0.23 \mathrm{eV}, 10^{-3}$ after $0.55 \mathrm{eV}$ and $10^{-4}$ after $1.04 \mathrm{eV}$, each of which is even better than comparable results [7] from literature. Based on this significantly improved experimental setup, first applications will be discussed. 


\section{References}

[1] A. Gloter et al., Ultramicroscopy 96 (2003) 385

[2] M. Rühle et al., Microsc. Microanal. 6, Suppl. 2: Proceedings (2000) 188

[3] F. Kahl, H. Rose, Proceedings 11th Europ. Cong. on Electron Microsc. Vol. 1 (1996) 478

[4] S. Uhlemann, M. Haider, Proceedings 15th Int. Cong. on Electron Microsc. Vol. 3 (2002) 327

[5] S. Uhlemann, H. Rose, Optik 96 (1994) 163

[6] E. Essers, G. Benner, Ultramicroscopy, submitted

[7] K. Kimoto et al., Micron 36 (2005) 185

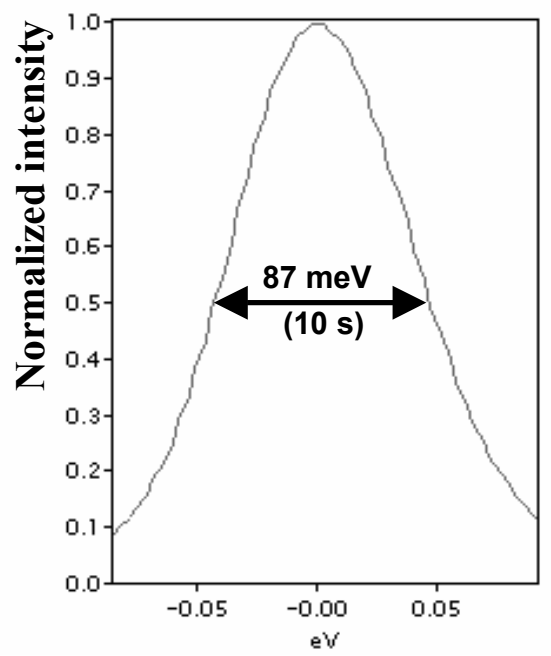

Fig.1: Zero-loss peak taken with $10 \mathrm{~s}$ exposure time. The maximum intensity is normalized to 1.

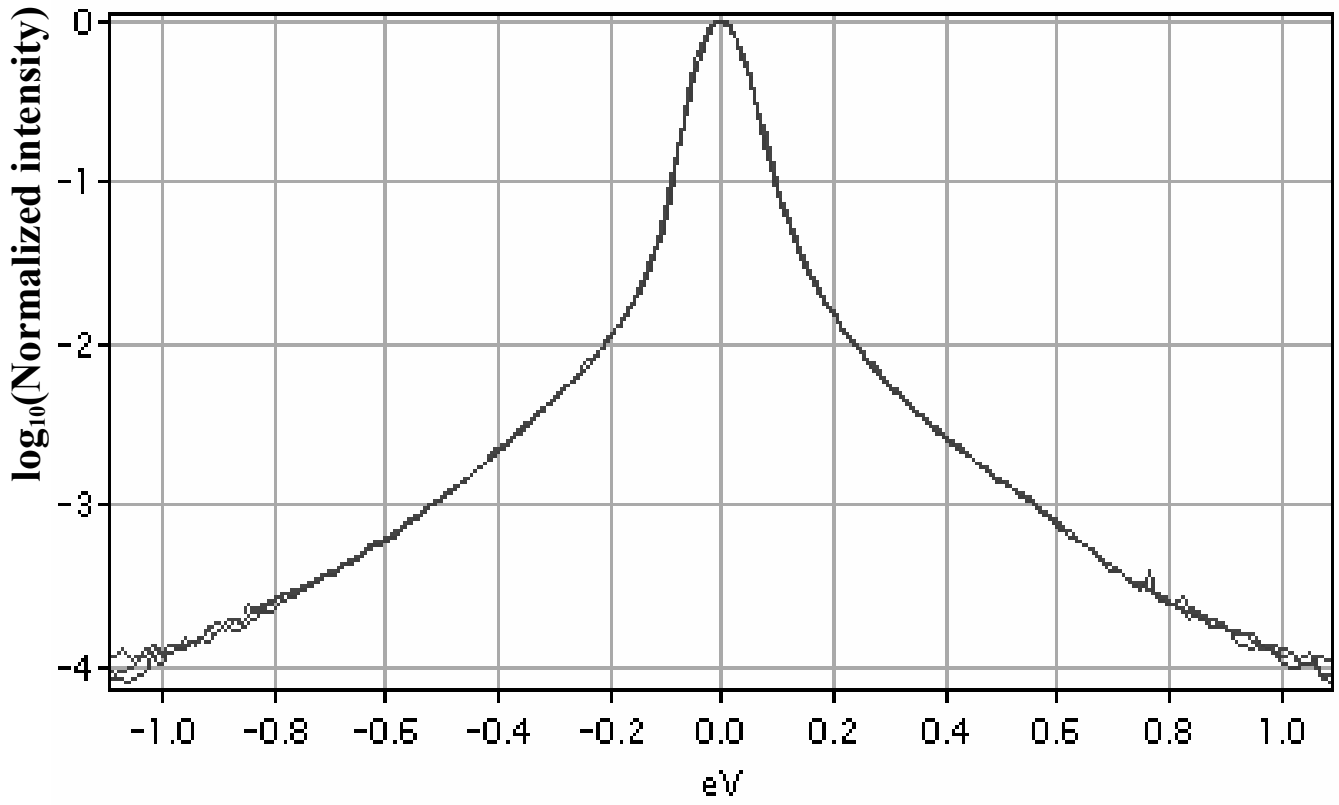

Fig.2: Three spectra of the zero-loss peak in logarithmic scale (taken with $10 \mathrm{~s}$ exposure time) showing the drop of the zero-loss peak and the reproducibility of its shape. 